04.2

\title{
Применение метода многочастотного допплеровского обратного рассеяния для исследования альфвеновских мод в токамаке
}

\author{
() В.В. Буланин ${ }^{1,2}$, В.К. Гусев ${ }^{1}$, Г.С. Курскиев ${ }^{1}$, В.Б. Минаев ${ }^{1}$, М.И. Патров ${ }^{1}$, \\ А.В. Петров ${ }^{2}$, Ю.В. Петров ${ }^{1}$, А.Ю. Яшин ${ }^{1,2}$ \\ ${ }^{1}$ Физико-технический институт им. А.Ф. Иофффе РАН, Санкт-Петербург, Россия \\ ${ }^{2}$ Санкт-Петербургский политехнический университет Петра Великого, Санкт-Петербург, Россия \\ E-mail: V.Bulanin@spbstu.ru
}

Поступило в Редакцию 15 июля 2019г.

В окончательной редакции 15 июля 2019г.

Принято к публикации 17 июля 2019 г.

\begin{abstract}
Приведены результаты исследования тороидальных альфвеновских мод с использованием многочастотного метода допплеровского обратного рассеяния в токамаке Глобус-М. Представлена методика регистрации альфвеновских мод при многоканальном зондировании. Детально обсуждаются возможные причины наблюдаемых колебаний полоидальной скорости вращения плазмы на частотах альфвеновских колебаний. Представлены сведения о пространственном распределении альфвеновских мод. Определены рекомендации для дальнейшего развития допплеровского обратного рассеяния с целью более детального исследования тороидальных альфвеновских мод в токамаках.
\end{abstract}

Ключевые слова: плазма, токамак, альфвеновская мода, допплеровское обратное рассеяние.

DOI: 10.21883/PJTF.2019.21.48474.17982

Исследование возникновения и развития альфвеновских мод, вызывающих потери высокоэнергетических ионов в токамаке, остается одной из основных экспериментальных задач в области реализации управляемого термоядерного синтеза и создания эффективных источников нейтронов [1-3]. Ранее сообщалось, что альфвеновские моды могут быть зарегистрированы методом допплеровского обратного рассеяния (ДОР) [4]. Использование этого метода открывает новую возможность изучения альфвеновских мод в центральных областях токамака. Эксперименты в работе [4] проводились в сферическом токамаке Глобус-М с использованием схемы ДОР, в которой зондирование плазмы осуществлялось СВЧ-излучением на одной фиксированной частоте, когда обратное рассеяние регистрировалось в ограниченном интервале по радиусу. Как показывает численное моделирование, в токамаке Глобус-М альфвеновские моды различных частот имеют неоднородное распределение по радиусу $[5,6]$. Для восстановления радиальных распределений требуется параллельная регистрация мод на различных радиусах. В настоящей работе для получения пространственных распределений альфвеновских мод в токамаке Глобус-М реализовано многочастотное зондирование плазмы.

Метод ДОР предназначен для регистрации мелкомасштабных флуктуаций плотности плазмы и определения скорости их вращения в направлении диамагнитного дрейфа заряженных частиц [7-10]. Скорость вращения $\mathbf{V}_{\perp}$ связана с величиной допплеровского смещения частоты обратно рассеянного излучения соотношением $\Delta \omega_{\mathrm{D}}=\mathbf{k}_{\perp} \mathbf{V}_{\perp}$, что позволяет определить величину и направление скорости вращения (здесь $\mathbf{k}_{\perp}-$ волновой вектор рассеивающих флуктуаций электронной плотности плазмы вблизи отсечки для случая распространения излучения О-моды). Допплеровское обратное рассеяние происходит на флуктуациях плазмы малых масштабов, в связи с этим рассеяние на альфвеновских модах, представляющих собой возмущения плазмы с поперечным размером порядка малого радиуса, не происходит. Тем не менее альфвеновские моды могут оказывать, как показано в работе [4], косвенное влияние на параметры обратно рассеянного излучения, которое выражается в появлении колебаний допплеровского частотного сдвига на альфвеновских частотах. Этот эффект использовался для регистрации альфвеновских мод при многочастотном зондировании. В настоящей работе для проведения исследований на токамаке Глобус-М использовалась четырехчастотная схема допплеровского обратного рассеяния [11]. Каждый частотный канал включал микроволновую схему с двойным гомодинным приемом, которая позволяла осуществлять квадратурное детектирование обратно рассеянного излучения. Использовались четыре фиксированные зондирующие частоты: 20, 29, 39 и $48 \mathrm{GHz}$. Зондирование плазмы осуществлялось со стороны слабого магнитного поля с помощью двух антенн с изменяющимся углом наклона относительно нормали к магнитной поверхности. Определение области обратного рассеяния проводилось с помощью ранее разработанного 3D-кода $[5,6]$. При выбранном наборе частот зондирующего излучения регистрацию альфвеновских мод можно было проводить в широком интервале по радиусу $\rho=0.6-1$ ( $\rho-$ нормированный малый радиус).

Применение четырехчастотной схемы допплеровского обратного рассеяния для регистрации альфвеновских 
мод осуществлялось в разрядах, в которых тороидальные альфвеновские моды надежно наблюдались в период инжекции пучка дейтерия с помощью магнитных зондов $[3,12]$. Основные характеристики разрядов были следующими: ток $I_{p}=170-250 \mathrm{kA}$, плотность плазмы $n_{e}<5 \cdot 10^{19} \mathrm{~m}^{-3}$, тороидальное магнитное поле $B_{T}=0.4$ и $0.5 \mathrm{~T}$. Энергия нагревного пучка дейтерия составляла $E_{b}=28 \mathrm{keV}$, мощность $P_{b}=0.75 \mathrm{MW}$. C помощью магнитных зондов в период стационарного плазменного тока через несколько миллисекунд после включения инжектора были зарегистрированы цуги колебаний на альфвеновских частотах, которые интерпретировались как тороидальные альфвеновские моды $[3,12]$. В ряде разрядов на спектрограммах сигналов магнитного зонда наблюдались вспышки альфвеновских мод, содержащие от одной до трех гармоник основной частоты. С использованием метода ДОР удалось зарегистрировать колебания допплеровского частотного сдвига $\Delta \omega_{\mathrm{D}}$ на альфвеновских частотах. Эти колебания наблюдались одновременно со вспышками колебаний сигналов магнитных зондов. Совпадали также частоты гармоник колебаний частотного сдвига и колебаний сигналов магнитного зонда. На рис. 1 показаны спектры магнитного зонда и спектры допплеровского частотного смещения для двух различных положений отсечки, вычисленные для одного и того же временно́го интервала длительностью $64 \mu \mathrm{s}$. Видно совпадение спектральных пиков на частоте $90 \mathrm{kHz}$ и ее гармониках в спектрах сигналов магнитного зонда и частотного сдвига. Было установлено (с использованием массива магнитных зондов), что различным гармоникам соответствуют альфвеновские колебания с различными тороидальными модовыми числами $n$. Различие в частотных спектрах допплеровского сдвига для положений отсечек $R_{\text {cutoff }}=51.8$ и $54.1 \mathrm{~cm}$ отражает различие в локализациях альфвеновских колебаний с разными модовыми числами $n$. Отметим, что пик на частоте $90 \mathrm{kHz}$ имелся и в спектрах колебаний амплитуды сигналов обратного рассеяния (рис. 1,d). Однако контраст этого пика значительно меньше, чем контраст пиков в спектрах колебаний допплеровского сдвига.

В работе [4] наблюдаемые колебания допплеровского частотного сдвига $\Delta \omega_{\mathrm{D}}$ на альфвеновских частотах связывались с колебаниями скорости рассеивающих флуктуаций $\mathbf{V}_{\perp}$. Полагалось, что эта скорость на альфвеновских частотах обусловлена $\mathrm{E} \times \mathrm{B}$ дрейфом в скрещенных радиальном электрическом поле альфвеновской волны $E_{r}$ и магнитном поле токамака. Электрическое поле альфвеновской моды ранее надежно регистрировалось с помощью метода зондирования пучком тяжелых ионов (НIBP) на стеллараторе TJ-II $[13,14]$. Основываясь на предположении о том, что скорость $\mathbf{V}_{\perp}$ есть скорость $\mathrm{E} \times \mathrm{B}$ дрейфа, можно оценить абсолютные значения амплитуды колебаний радиального электрического поля $\tilde{E}_{r}=\tilde{V}_{\perp} B$ и использовать полученные данные для нахождения амплитуды колебаний полоидальной проекции магнитного поля в альфвеновской электромагнит-

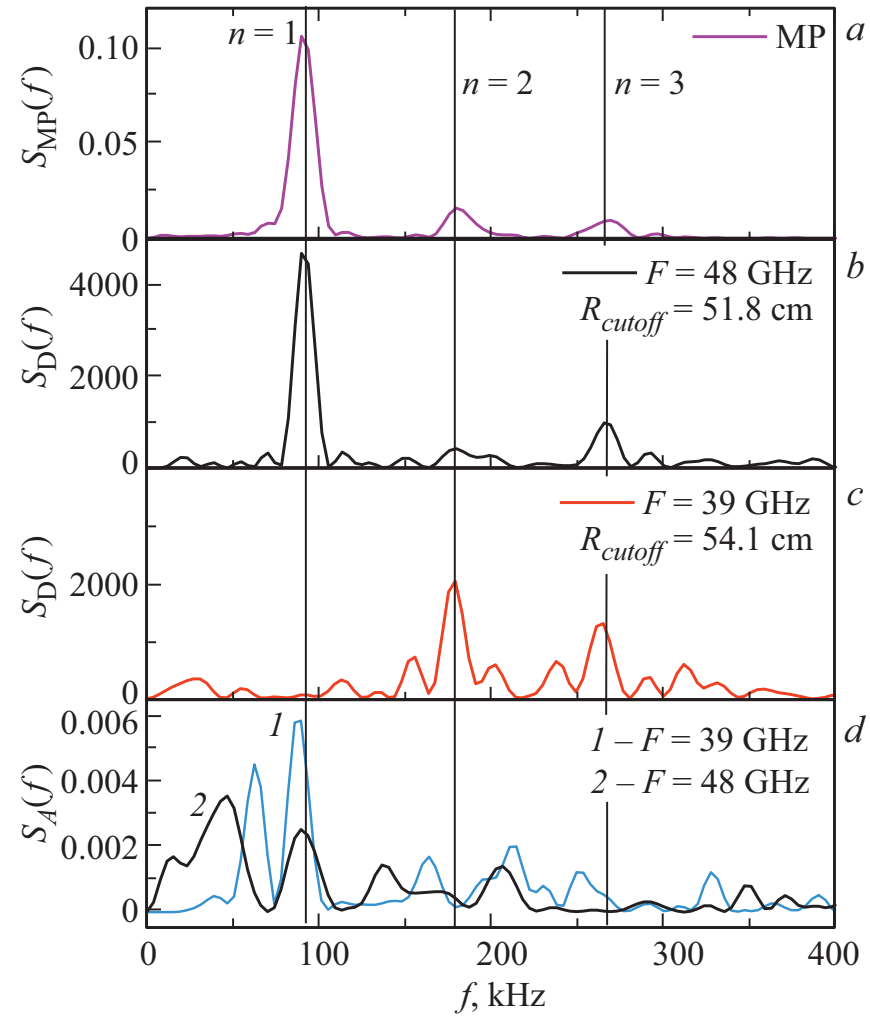

Рис. 1. Спектры мощности сигналов магнитного зонда $(a)$, допплеровского частотного сдвига $(b, c)$, модуля сигнала IQ-детектора $(d)$. Выстрел токамака \#36994, момент времени $t=146.2 \mathrm{~ms}, F-$ частота зондирования.

ной волне $\tilde{B}_{\theta}$. Определенные таким образом значения отвечают локальным величинам полей в области отсечек зондирующего излучения. С учетом рассеяния излучения различных СВЧ рассчитанные параметры находились в следующих пределах: $V_{\theta}=2-9 \mathrm{~km} / \mathrm{s}$, $E_{r}=0.5-3 \mathrm{kV} / \mathrm{m}, B_{\theta}=(7-25) \cdot 10^{-4} \mathrm{~T}$. Колебания магнитного поля в альфвеновской волне приводят к деформации магнитных поверхностей, которая вызывает вариацию угла падения излучения и колебания с альфвеновской частотой вектора $\mathbf{k}_{\perp}$. Изменения $\mathbf{k}_{\perp}$ в свою очередь могут приводить к колебаниям $\Delta \omega_{\mathrm{D}}$ [4]. Однако амплитуда этих колебаний $\Delta \omega_{\mathrm{D}}$ по оценкам значительно меньше амплитуды, наблюдаемой в эксперименте. Поэтому с учетом новых данных об амплитуде колебаний магнитного поля, полученных при многочастотном зондировании, можно полагать, что колебания скорости это колебания скорости дрейфа в радиальном электрическом поле. Колебания $\mathbf{k}_{\perp}$ могут также вызывать колебания амплитуды рассеивающих флуктуаций при их сильной зависимости от волнового числа. Наблюдаемые малые колебания интенсивности рассеянного излучения, которые проявляются в спектральных пиках на спектрах (рис. $1, d)$, возможно, связаны с этим эффектом.

С использованием всего массива данных, полученных при многочастотном зондировании, было проведено 


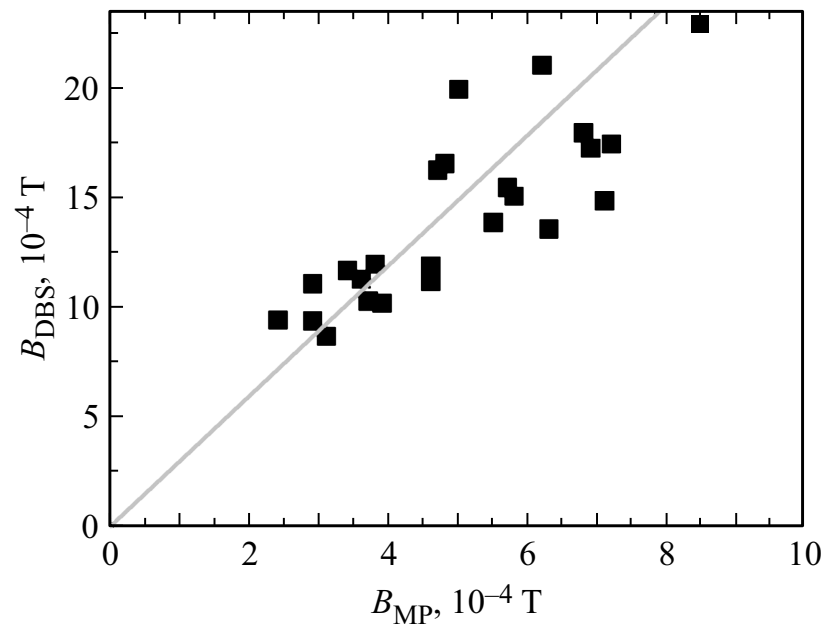

Рис. 2. Сопоставление амплитуд колебаний полоидальной компоненты магнитного поля альфвеновской волны в плазме, измеренных с помощью метода допплеровского обратного рассеяния $\left(B_{\mathrm{DBS}}\right)$ и с использованием магнитного зонда вне разряда $\left(B_{\mathrm{MP}}\right)$.

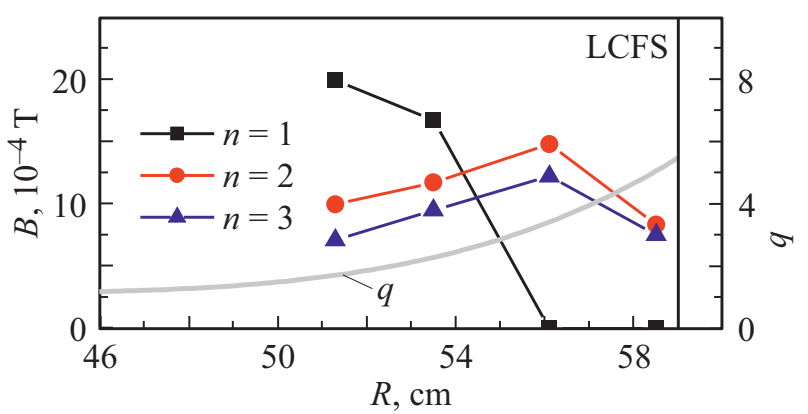

Рис. 3. Распределение амплитуд колебаний магнитного поля для различных тороидальных модовых чисел $n . q-$ коэффициент запаса устойчивости, LCFS - последняя замкнутая магнитная поверхность, разряд токамака \#36988, $I_{p}=236 \mathrm{kA}$, $t=147.6 \mathrm{~ms}$.

сравнение амплитуд колебаний магнитного поля, изме-

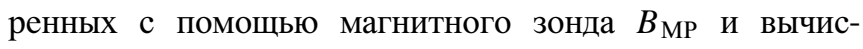
ленных с использованием данных диагностики допплеровского обратного рассеяния $B_{\mathrm{DBS}}$. Магнитный зонд, расположенный на радиусе $R=62.5 \mathrm{~cm}$, регистрировал колебания полоидальной составляющей магнитного поля. Результаты сравнения представлены на рис. 2. Значения, определенные с помощью периферийного магнитного зонда, оказывались меньше в силу естественного спада магнитного поля с увеличением расстояния от источника колебаний. При этом прослеживается пропорциональная зависимость между величинами магнитных полей $B_{\text {MP и }} B_{\text {DBS. }}$.

Основное назначение многочастотного метода обратного допплеровского рассеяния заключалось в определении интенсивности альфвеновских мод одновременно на четырех радиусах, что позволяло восстановить пространственное распределение колебаний магнитного поля для различных значений тороидального модового числа $n$ для альфвеновских мод. В качестве примера на рис. 3 представлены распределения амплитуд одного из цугов альфвеновских колебаний, наблюдаемых в разряде \#36988. Как видно из рис. 3, альфвеновские моды с тороидальными волновыми числами $n=2$ и 3 были локализованы на периферии плазмы. Что касается колебаний с модовым числом $n=1$, то отмечалось смещение радиального распределения амплитуд колебаний с $n=1$ в центральную область разряда с ростом плазменного тока и уменьшением запаса устойчивости на границе плазмы.

Таким образом, многочастотное допплеровское обратное рассеяние позволило определить пространственные распределения альфвеновских мод, которые позволят провести сопоставление с результатами численного моделирования альфвеновской неустойчивости в токамаке Глобус-М. Эксперимент указал на необходимость расширения диапазона зондирующих частот и увеличения числа каналов с целью регистрации альфвеновских колебаний в центральных областях разряда с лучшим пространственным разрешением.

\section{Финансирование работы}

Эксперименты проведены на УНУ „Сферический токамак Глобус-М“ в рамках государственного задания Минобрнауки РФ. Исследование тороидальных альфвеновских мод выполнено при поддержке Российского научного фонда (грант № 17-12-01177).

\section{Конфликт интересов}

Авторы заявляют, что у них нет конфликта интересов.

\section{Список литературы}

[1] Heidbrink W.W. // Phys. Plasmas. 2008. V. 15. P. 055501.

[2] Gorelenkov N.N., Pinches S.D., Toi K. // Nucl. Fusion. 2014. V. 54. P. 125001.

[3] Petrov Yu.V., Bakharev N.N., Gusev V.K., Minaev V.B., Kornev V.A., Kurskiev G.S., Patrov M.I., Sakharov N.V., Tolstyakov S.Yu., Shchegolev P.B. // J. Plasma Phys. 2015. V. 81. P. 515810601.

[4] Буланин В.В., Гусев В.К., Курскиев Г.С., Минаев В.Б., Патров М.И., Петров А.В., Петров М.А., Петров Ю.В., Тельнова А.Ю., Яшин А.Ю. // Письма в ЖТФ. 2017. Т. 43. C. 40-47.

[5] Medvedev S.Yu., Martynov A.A., Gusev V.K., Petrov Yu.V., Patrov M.I., Tel'nova A.Yu., Ivanov A.A., Poshekhonov Yu.Yu. // ВАНТ. Сер. Термоядерный синтез. 2018. Т. 41. B. 2. C. $95-104$.

[6] Петров Ю.В., Бахарев Н.Н., Буланин В.В., Гусев В.К., Курскиев Г.С., Мартынов А.А., Медведев С.Ю., Минаев В.Б., Патров М.И., Петров А.В., Сахаров Н.В., Щеголев П.Б., Тельнова А.Ю., Толстяков С.Ю., Яиин А.Ю. // Физика плазмы. 2019. Т. 45. В. 8. С. 675-684.

[7] Bulanin V.V., Lebedev S.V., Levin L.S., Roytershteyn V.S. // Plasma Phys. Rep. 2000. V. 26. P. 813-819. 
[8] Hirsch M., Holzhauer E., Baldzuhn J., Kurzan B., Scott B. // Plasma Phys. Control. Fusion. 2001. V. 43. P. 1641-1660.

[9] Conway G.D., Schirmer J., Klenge S., Suttrop W., Holzhauer E. and the ASDEX Upgrade Team // Plasma Phys. Control. Fusion. 2004. V. 46. P. 951-970.

[10] Hennequin P., Honoré C., Truc A., Quéméneur A., FenziBonizec C., Bourdelle C., Garbet X., Hoang G.T. and the Tore Supra Team // Nucl. Fusion. 2006. V. 46. P. S771-S779.

[11] Yashin A.Y., Bulanin V.V., Petrov A.V., Petrov M.A., Gusev V.K., Khromov N.A., Kurskiev G.S., Patrov M.I., Petrov Y.V., Tolstyakov S.Y., Prisyazhnyuk D.V. // JINST. 2015. V. 10. P. P10023.

[12] Бахарев Н.Н., Гусев В.К., Ибляминова А.Д., Корнев В.А., Курскиев Г.С., Мельник А.Д., Минаев В.Б., Патров М.И., Петров Ю.В., Сахаров Н.В., Толстяков С.Ю., Хромов Н.А., Чернымев Ф.В., Щёголев П.Б., Вагнер Ф. // Письма в ЖТФ. 2013. Т. 39. В. 24. С. 22-29.

[13] Melnikov A.V., Eliseev L.G., Ascasibar E., Chmyga A.A., Hidalgo C., Ido T., Jiménez-Gómez R., Komarov A.D., Kozachek A.S., Krupnik L.I., Khrebtov S.M., Könies A., Kuznetsov Yu.K., López-Fraguas A., Lysenko S.E., Mavrin V.A., Nagaoka K., de Pablos J.L., Pedrosa M.A., Perfilov S.V., Smolyakov A.I., Spong D.A., Ufimtsev M.V., Yamamoto S. and the TJ-II Team // Nucl. Fusion. 2012. V. 52. P. 123004.

[14] Melnikov A.V., Ochando M., Ascasibar E., Castejon F., Cappa A., Eliseev L.G., Hidalgo C., Krupnik L.I., LopezFraguas A., Liniers M., Lysenko S.E., de Pablos J.L., Perfilov S.V., Sharapov S.E., Spong D.A., Jimenez J.A., Ufimtsev M.V., Breizman B.N., HIBP group and the TJ-II Team // Nucl. Fusion. 2014. V. 54. P. 123002. 